\title{
The Default Space Theory of Consciousness: Phenomenological Support from Personal Observations and Clinical Deficits
}

\author{
Ravinder Jerath $^{1 *}$, Connor Beveridge ${ }^{1}$, Michael Jensen ${ }^{2}$ \\ ${ }^{1}$ Charitable Medical Healthcare Foundation, Augusta, GA, USA \\ ${ }^{2}$ Augusta University, Augusta, GA, USA \\ Email: *Rj605r@aol.com
}

How to cite this paper: Jerath, R., Beveridge, C. and Jensen, M. (2019) The Default Space Theory of Consciousness: Phenomenological Support from Personal Observations and Clinical Deficits. World Journal of Neuroscience, 9, 1-21. https://doi.org/10.4236/wjns.2019.91001

Received: November 29, 2018

Accepted: January 11, 2019

Published: January 14, 2019

Copyright $\odot 2019$ by author(s) and Scientific Research Publishing Inc. This work is licensed under the Creative Commons Attribution International License (CC BY 4.0).

http://creativecommons.org/licenses/by/4.0/

\begin{abstract}
Consciousness is the unified, structured, subjective experience that we all share. The Default Space Theory has been proposed as a unified theory of consciousness that includes the brain and body in describing the infrastructure of consciousness. We have presented the theory in journals with a variety of academic specialties; however, the model is continually being developed. Due to the current state of science on the nature of conscious experience lacking hard data, the theory's concepts must thoroughly explain and shoulder phenomenological observations including the phenomenology of deficits of consciousness. According to the theory, the thalamus serves as a central hub which networks the globally distributed, and continuous fast oscillations not only among the brain, but also the eyes, ears, skin, and other sensory organs. These oscillations form the virtual template of external space within the mind in which external sensory information is integrated into this pre-existing, dynamic space. In this article, we explore phenomenological support for our theory of conscious experience in which such experience resides entirely in such a virtual space, termed the default space. In order to provide such support, we discuss simple personal experiments and observations which anyone can partake along with phenomenal symptoms of clinical deficits. We encourage readers to perform the personal experiments we describe in order to gain an understanding of the various concepts of the theory. The neurological deficits we elucidate not only support the theory but clarify obscurities surrounding these conditions. We assert the support we give in our theory here will advance the uphill struggle many paramount theories face in gaining initial acceptance. Further research is needed in order to acquire empirical evidence for the veracity of our theory.
\end{abstract}




\section{Keywords}

Default 3D Space, Thalamus, Consciousness, Corticothalamic Feedback Loop, Contralateral Neglect, Phantom Limb, Lateral Inhibition

\section{Introduction}

We define consciousness simply in terms of the presence of experience. Perhaps the most difficult problem in science is the "hard problem of consciousness" [1] [2]. How do the qualia of experience arise from the strictly physical, electrical, and chemical physiology of life? How does the simple experience of colors or shapes come about from atoms jostling around in the large ordered clump we call a human body? Many hypotheses have been formed in academic areas in an attempt to scratch away at this hard problem [3] [4]; however, these hypotheses fail to unify the many aspects of consciousness as we know it in the human mind. We have previously proposed a unified theory of consciousness, the Default Space Theory (DST) [5]; what we propose may provide a means to one day solving this problem which although may be out of the reach of human understanding all together [6]. We assert that the DST elucidates a more realistic problem to solve, the "real problem" of consciousness that ponders the various characteristics of consciousness in terms of biological mechanisms instead of questioning the physical nature of its existence in the universe [7].

Our theory offers insight into this "real problem" by describing the physiologically unified nature of consciousness including its various states such as the coma, sleep, and awake states [5]. We have and continue to support the DST by pointing to evidence from a wide range of areas including anatomy, cellular biology, electrophysiology, simple self-experimentations, clinical neurological deficits, neuroscience, psychology, and evolution. Since the initial publication on our theory, we have further developed and revised our hypotheses and continue to detail various disorders it explains such as central pain syndrome [8], contralateral neglect syndrome, dizziness, claustrophobia [9], and phantom limb syndrome [10]. Phenomenology is the study of the structure of conscious experience. We review the phenomenology of these deficits along with several newly proposed personal observations that allow the layperson to have a glimpse of the structure and dynamic nature of mind. When we contemplate consciousness through the lens of our own experience along with scientific research, we can truly begin to understand it.

\section{The 3D Default Space}

The DST describes how sensory information is processed, integrated, unified, and filled-in within a 3D (three-dimensional) intra-personal space which is the bare framework of consciousness [11]. Such a 3D spatial matrix or a general spatial structure has been proposed by other leading consciousness researchers as 
the phenomenal axiom of consciousness [12] [13]. This space contains what is essentially an internal simulation of the surrounding physical environment (including one's own bodily sensations) [14], and could even be considered an informed hallucination [15]. All conscious experience, including dreaming and recollection of memories, occurs within this default space [16]. Because the default space mimics the physical nature of the environment, it allows for optimal interactions with the environment. We assert this evolutionary benefit lead the ancient, "prototype", intelligent biological structures of human and animal ancestors to develop into minds utilizing some form of a default space [11]. This evolutionary development may have its origins in the first conscious organisms.

In contrast to other similar consciousness models which view consciousness as arising soley from the brain [17], the default space is comprised from all the cells of the body [16], which are all connected via afferent and efferent neurons [18], gap junctions, and electrical membrane potentials [11]. The DST posits the thalamus as the central hub of the default space with 50 or more integrated nuclei coordinated by the reticular nucleus [11]. This central hub acts as a tiny "observer" of the large electro-chemical space formed by the trillions of cells of the body. The processing of the bodily signals through the corticothalamic feedback loops is not complete until these signals become filtered, amplified, and integrated by the varied cortical modules. A unique principle of the DST is that these processed signals return to the peripheral sensory and visceral organs in a top-down dominated fashion via lateral inhibition [19] [20]. In this manner, expectations about reality can be formed and incoming sensory information can simply "fall into place", allowing for a near real time experience of the internal and external world [21]. Although it may appear as if we are experiencing the external world directly, we are in reality generating an internal representation of it heavily influenced by memories, understandings about the physical world, and expectations [14].

We have explained how fast gamma oscillatory communications between peripheral receptors such as photoreceptors exhibit "lateral inhibition across abstract dimensions", allowing the corticothalamic networks to interact with the retina (or other sensory organ) and "view" it as if it were a 3D "Smart Screen" [20]. The currently accepted view in science is that this universal physiology is local and "lateral" [22]. When we speak of lateral inhibition, we do not mean in the commonly used sense indicating cells inhibiting physically adjacent cells, however, cortical inhibitions of sensory organ cells that are "adjacent" via synchronization. Although the cortex is not physically adjacent to the sensory organs, it is functionally adjacent due to the synchronization of said cortex with such sensory organs. This allows communication at near the same time interval as if the cells where physically adjacent. This fast time interval is possible at such separated distances due to the speed of charge transmission. We do, however, note that receptors synchronized with the cortex laterally inhibit its physical neighbors, although the main source of processing determining how cells are inhibited comes from the cortex. This can lead to a magnification of sensory sig- 
nals, and/or a filtering of unwanted data.

\section{Personal Tests and Observations to Substantiate the Default Space Theory}

Scientific history is filled with breakthrough theories that struggled to become accepted [23]. For instance, the theories of Darwin, Prusiner, and Einstein required protracted uphill battles before they became the scientific foundations we know today [23]. We have proposed numerous tests that give support for the DST, with confidence that more modern tests will solidify our theory as the paramount theory of consciousness. Aside from the analysis of various neurological illnesses, there are many simple tests and observations that can be performed by the layperson which may not only substantiate the DST, but provide personal insight into mechanisms of consciousness. We explore these tests and encourage readers to perform them. These tests not only highlight the nature of the idling default space and how it is filled with sensory information, but the phenomena described also explore the fringes of the model. Through close examination of one's personal phenomenology, mechanisms of consciousness may be revealed [13].

\subsection{Rubber Hand Test}

The rubber hand illusion demonstrates the top-down predominance of perception which begins with the placement of a fake hand in close proximity and orientation to an observer's concealed real hand [24]. Observing the rubber hand being touched synchronously with the observers own unseen hand often causes the observer to associate the rubber hand to the observer's own body, taking the place of the real hand [25]. A perceptual drift occurs from the normal perception of one's own hand toward the experience of the rubber hand as one's real hand. This phenomenon is also present in the full body illusion, in which the participants in a similar manner come to identify a virtual body, not just a hand, as their own [26]. This effect can be explained in part by bottom-up processes of visuo-tactile correlation driving the development of top-down processes that determine body schema [25]. It is interesting to note that upon disowning one's hand, physiological changes occur in that hand with studies showing temperature decreases in the disowned limb [27]. This indicates that consciousness of our embodiment is linked to physiological regulation of our bodies.

This test supports the DST's concept of intra-personal space being a flexible representation of the body schema and contents. The DST posits that body schema arises from constantly adjusting representations of internal and external space [10]. The rubber hand test highlights the prominence of vision and subconscious expectations over non-visual sensory input in determining what is filled into the default space, as well as supporting the top-down dominance of sensory experience and the experience of the self. This test further supports the DST's assertion that we don't experience reality as direct sensory signals, but 
however develop a most accurate simulation of reality internally which integrates external percepts with proprioceptive analyses of embodiment into a unified, cohesive experience of reality with oriented self-boundaries all based in and upon the three dimensional framework of the known universe.

\subsection{Dizziness Test}

After spinning around multiple times to produce dizziness, one will notice the surrounding environment continues to spin upon stopping. The continued spinning of the visual scene is due to the combination of variant visual and vestibular sensory information integrated into the 3D default space [28]. Once the person stops spinning, the visual scene becomes static; however, fluids continue to move within the semicircular canals of the inner ear [29]. The continued movement of the non-visual space indicated from the vestibular organs creates the sensation of a continued spin, and this vestibular non-visual sensory space is integrated with the visual sensory space. Although the brain combines the two streams of information into one virtual 3D space which is experienced as a unified event, the integration conflict between the two senses naturally leads to the dizziness and nausea [28]. When spinning with eyes closed, less sensory conflict is present and so less nausea is experienced, however one may continue to feel that they are spinning around upon stopping as the vestibular organs indicate such a state.

This test demonstrates the process of integration and unification of sensory information into the default space. Because consciousness is a unified experience, the brain must make compromises when incoming sensory information to be integrated does not agree. In the case of dizziness, this compromise takes the form of combining the visual scene with the spinning motion indicated by the vestibular organs. The $3 \mathrm{D}$ orientation one maintains in respect to the thalamic viewpoint is therefore thrust into the spinning motion as the vestibular senses dominate this perspective. Even when one spins with eyes closed, dizziness may still be experienced as there is a conflict between bottom-up information from the vestibular organs and the top-down expectation that one has ceased to spin. We propose that if someone is spun around with eyes remaining closed and upon stopping is somehow convinced that they are still spinning, no dizziness will be experienced as no sensory conflicts are present.

\subsection{Positive Afterimage Test}

A traditional positive afterimage is an optical illusion that results in an image continuing to be visible after it has left the viewer's current visual scene. The common experience of an afterimage of a bright scene upon closing the eyes has been proposed to be due in part to sensitization changes of photoreceptors [30]; however, studies have shown that the cortex is also involved in the appearance of these afterimages [31] [32] [33]. We have proposed that the internal visual representation of the scene has a major role in the presence of afterimages [34] 
(Figure 1). This simple observation indicates that we have an internal 3D visual space, which we have proposed is maintained by default activity of retinogeniculo-cortical oscillations [19]. Even when the eyes have been closed, this 3D visual space remains intact with chemical memories on the photoreceptors along with correlations in the cortex keeping the last image seen in this visual space in the same orientation to the thalamic viewpoint even as our true orientation changes.

\subsection{Negative Afterimage Test}

A positive afterimage results in a similar colored image remaining in sight. Similarly, a negative afterimage results in an image remaining in sight after it has left view, however, the resulting image is the negative of the original. Negative images have brightness reversed as well as colors becoming that of their complementary color. The consensus has been that this is due to rods and cones adapting to overstimulation with resulting sensitivity changes [35]; however, newer evidence is showing that there is cortical involvement [31]. We have proposed a new etiology for this phenomenon and while sensitization changes may account for some of the negative afterimage, we assert that the full experience is explained by mechanisms of the ON-center/OFF center cones of the retina [19] [36].

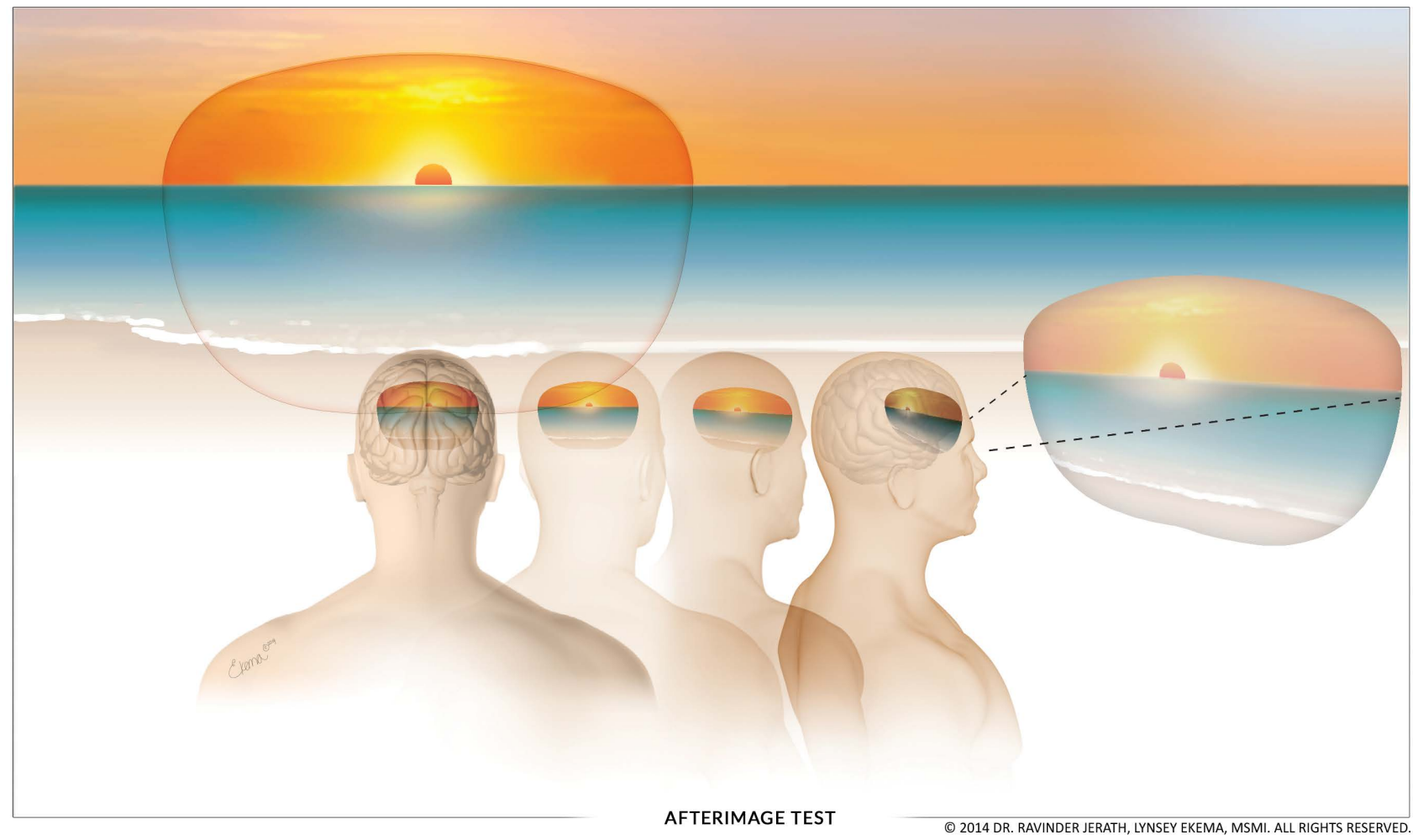

Figure 1. The Afterimage Test: The figure illustrates the nature of an afterimage with a person initially staring out into the sunset. Upon looking away from the sunset at any angle with eyes closed or in a dark space, a faded and perhaps blurry original image of the sunset is visible on the back of the eyelids or in the current dark visual scene. As the head is rotated, the afterimage follows. We suggest that this afterimage is a remnant of the external space defaulted into the internal space with filling in by higher cortical areas and the retina [28]. 
Our conjecture has been that negative afterimages begin with millions of on-center cones receiving the initial visual stimuli and naturally registering the information as opposite colors of the incoming transduced light, and this is where the opposite colors of the negative afterimage originate [19]. Due to the synchronization of the retina with the cortex, this information is processed immediately and the transduced light data combined with associated synchronized input from the thalamus and cortex is then amplified onto the OFF-center cones through lateral inhibition as the actual color experienced [19]. It is this amplified image that dominates the visual qualia filled into the default space. Negative after-images result from the initial electro-chemical signal on the ON-center cones which remain longer than the image amplified by the OFF-center cones as well as lacking amplification leading to a faint image [19]. When we look at a blank white canvas after staring at a color image, we see its color as inverse because the ON-center cones have not "recharged", and the rhodopsin in them is still signifying an inverse color. This inverse color is still filled into the default space due to the synchronization between the retina and cortex as well as the mechanisms of visual consciousness occurring on the retina itself. Because the ON-center cone qualia are much weaker than the OFF-center cone qualia, the negative afterimage can only be seen upon a uniform background such as a white canvas.

\subsection{Bright Light Test}

This test begins with visual exposure to a bright light source. With eyes closed, a bright red glow is seen. When a hand is placed in front of one of the closed eyes the glow is halved and it resembles the shape of a single visual field. This test helps visualize the invisible separation of the visual fields, the structure of the internal visual space, and the nature of sensory integration into the default space [34]. The reddish glow in the shape of a single visual field indicates that we are viewing the external visual space as if from the surface of the retina. If the visual field is experienced inside the skull, there would logically be a round glow in the shape of a round eye. The process is described in detail in recent publications on the retina [19].

\subsection{Meditation Experiences}

Meditation is practiced globally by millions of people including 18 million in the U.S. [37]. Benefits of meditation have been discussed and reported first hand and experimentally [38]. Positive physiological and psychological changes that result from regular meditation practice have been shown to perpetuate well-being and improved cognitive faculties such as learning ability [39]. The experience of pure consciousness during the practice of Transcendental Meditation is one of "self" awareness or "self" referral [40]. Reportedly, advanced practitioners of various meditation techniques experience unbounded space and time [41], supporting our theory that the default space is a borderless intrapersonal space and is what meditators become more aware of during their sessions [42]. Benefits of meditation are reflected in the phenomenological changes in prac- 
ticed meditators. Aside from increased joy, meditators experience increased perceptual vividness and intensity and increased self-awareness [43].

\subsection{The Invisible Gorilla Test}

The DST is not the first psychological rumination to assert the strong association between attention and consciousness, evident in the popular psychological demonstration referred to as the "Invisible Gorilla Test" [44]. In this test of attention and consciousness, a person who has not previously heard of the test is shown a video of some young people tossing around a basketball [45], and is instructed to focus their attention on counting how many times the ball is passed. During the middle of this series of ball passes, someone in a gorilla suit dances through the middle the group. Astoundingly, the majority of the viewers who were counting the ball tosses do not consciously notice the gorilla. This is clear evidence that attention is required for sensory information to reach consciousness, supporting the DST's analysis of various layers of consciousness [46]. This test also demonstrates how the default space replicates the external world into the internal, but heavily filters out unnecessary sensory data through involvement of executive faculties so one can best focus on the task at hand. We assert that due to the top-down dominance of sensory expectation, if we don't expect to see the gorilla and don't pay attention to it, the visual sensory data of the gorilla will not be amplified from the ON-center photoreceptors onto the OFF-center ones, and therefore will not be projected into the default space to be experienced. This further demonstrates the dynamic relationship between executive functions and the sensory organs.

\subsection{The Ganzfeld Effect and Dreaming}

The previously proposed dark space is the idling foundation of the default space [42]. This dark space is the infrastructure of consciousness, ready to instantly be filled with qualia derived from the external world [42]. The Ganzfeld effect illustrates the presence of this space. Perceptional deprivation: exposure to a uniform, unstructured sensory field, leads to the hallucinatory phenomenon of the Ganzfeld effect [47]. Similar effects arise from perceptual isolation, which has been used for meditation [48], as well as torture [49]. The difference is that meditation is self-induced, not forced. When sensory cortical areas are deprived of sensory information for an extended period, they become easily excitable [48]. These excited cortical areas actively search for novel sensory information and attention is drawn to internally generated neural noise and percepts, resulting in hallucinations [50]. Although the cortical sites essentially produce their own sensory activity instead of receiving it, this experience is seemingly real because this activity is still filled into the dark space in which it is experienced. One can experience the Ganzfeld effect by 1) cutting a ping pong ball in half, 2) placing each half over the eyes, and 3) turning on the radio to static [50].

A similar sensation-producing event occurs during dreaming. During a dream 
state a person will experience the same external space within the mind, however its contents are created by the mind and the dreamer will experience the dream as if they were awake [51]. The phenomenon of dreaming shows that without any external sensory input, the intra-personal default space is still active and imparts the feeling of external space. Upon recollection of a scene from memory, the experience will still occur in the default space with objects, feelings, and the space of the scene functionally represented in the default space [11]. We assert that these phenomena help support the existence of the default space through correlating all conscious experience to this space. When we hallucinate, dream, imagine, or remember, sensory qualia originating from whatever cortical activity is producing it are integrated into this $3 \mathrm{D}$ inner space, and therefore have a spatial component. We further propose that when we imagine for instance the color red, top-down projections actually send sensory information indicating the color red to our retina where it appears on the photoreceptors. This demonstrates the dynamic relationship between what we experience in the default space with the sensory organs we possess.

\section{Etiology of Neurological Conditions}

The DST has proposed novel etiologies for neurological conditions and syndromes that affect consciousness in some way. We assert that any illness of consciousness can be explained through the DST as it encompasses the framework of consciousness. We will revisit novel etiologies of various conditions with new insights in light of our model. Each of the syndromes discussed here will focus on how the model is supported by these conditions. Not only are novel etiologies explained, but the respective deficits of mind also provide evidence for the veracity of the DST of consciousness.

\subsection{Contralateral Neglect Syndrome}

Patients with lesions in the right parietal lobe may develop contralateral neglect syndrome and be unable to see or locate objects within the left external visual and non-visual space [52] (Figure 2, Figure 3). Support for the DST has been drawn from the observation that in those with contralateral neglect syndrome, not only is sensory information from the left side of the external space lost, but sensation from the intra-personal space is lost as well [51]. This syndrome supports the DST's keystone principle that 3D space is the foundation of sensation and experience. Spatial sensory information for the left side of this 3D space is lost; however, patients do not experience any sensation whatsoever on the left side of their perspective [51]. One can logically conclude that the internal representation of 3D space must be intact before one can experience the internal recreation of the external world [11].

\subsection{Phantom Limb Syndrome}

Phantom limb pain exists in those who had a body part amputated or paralyzed, 


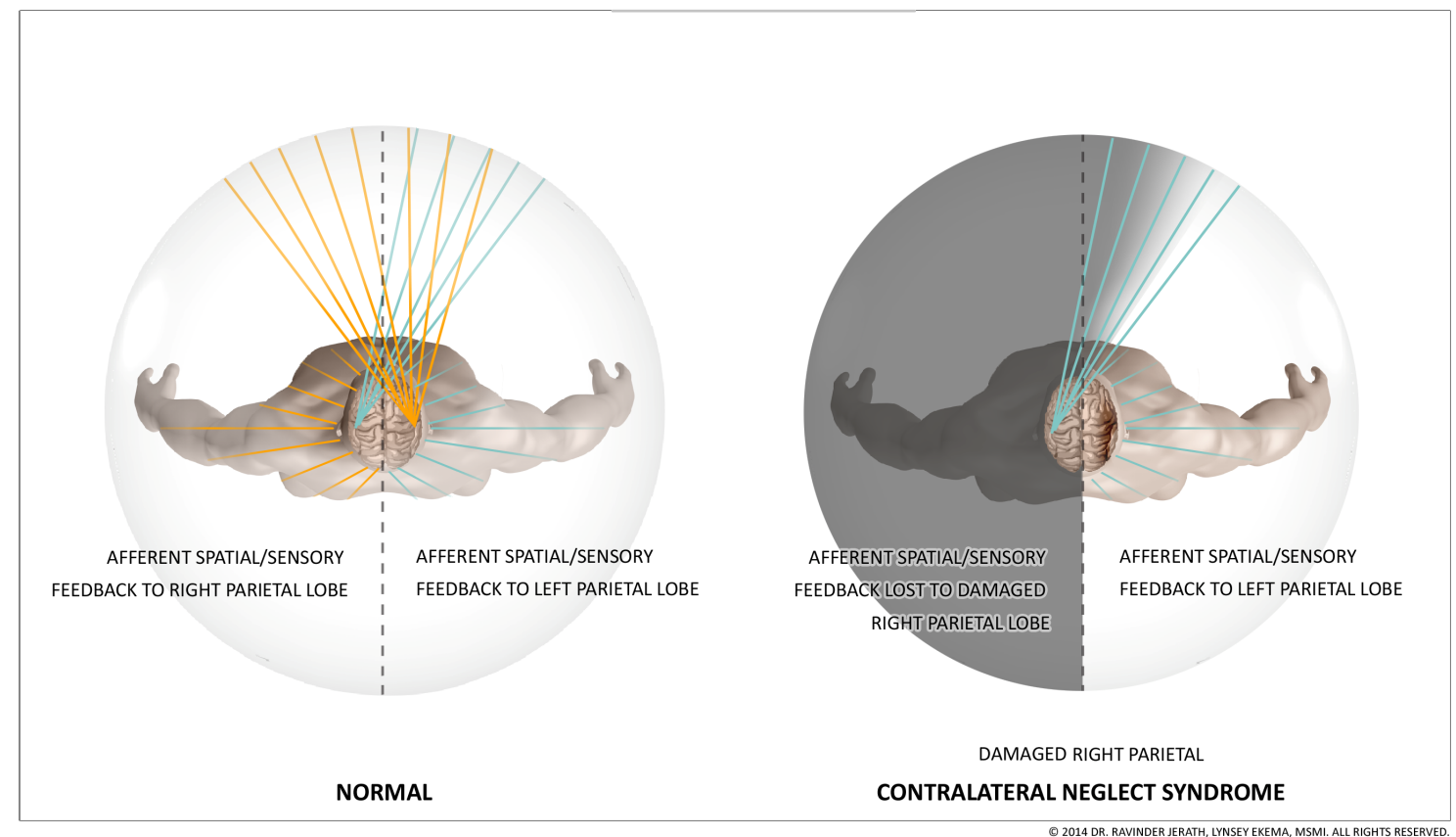

Figure 2. Connections of the left and right parietal cortex: visual fields and body space under contralateral neglect. The figure depicts how the right parietal lobe in a healthy individual acquires sensory information from the left side of the body while the left parietal lobe does so with the right side of the body. The parietal lobes spatially map sensory information. In contralateral neglect syndrome, the right parietal lobe is damaged leading to the loss of the sensory information fed to it. This lost information does not become spatially oriented, therefore, never becomes a part of conscious awareness within the 3D default space. This results in a complete lack of awareness of stimuli on their left side, even though the peripheral nervous system is functioning normally (Figure and figure description reproduced with permission from copyright holder; Figure by Lynsey Ekema, MSMI) [53].

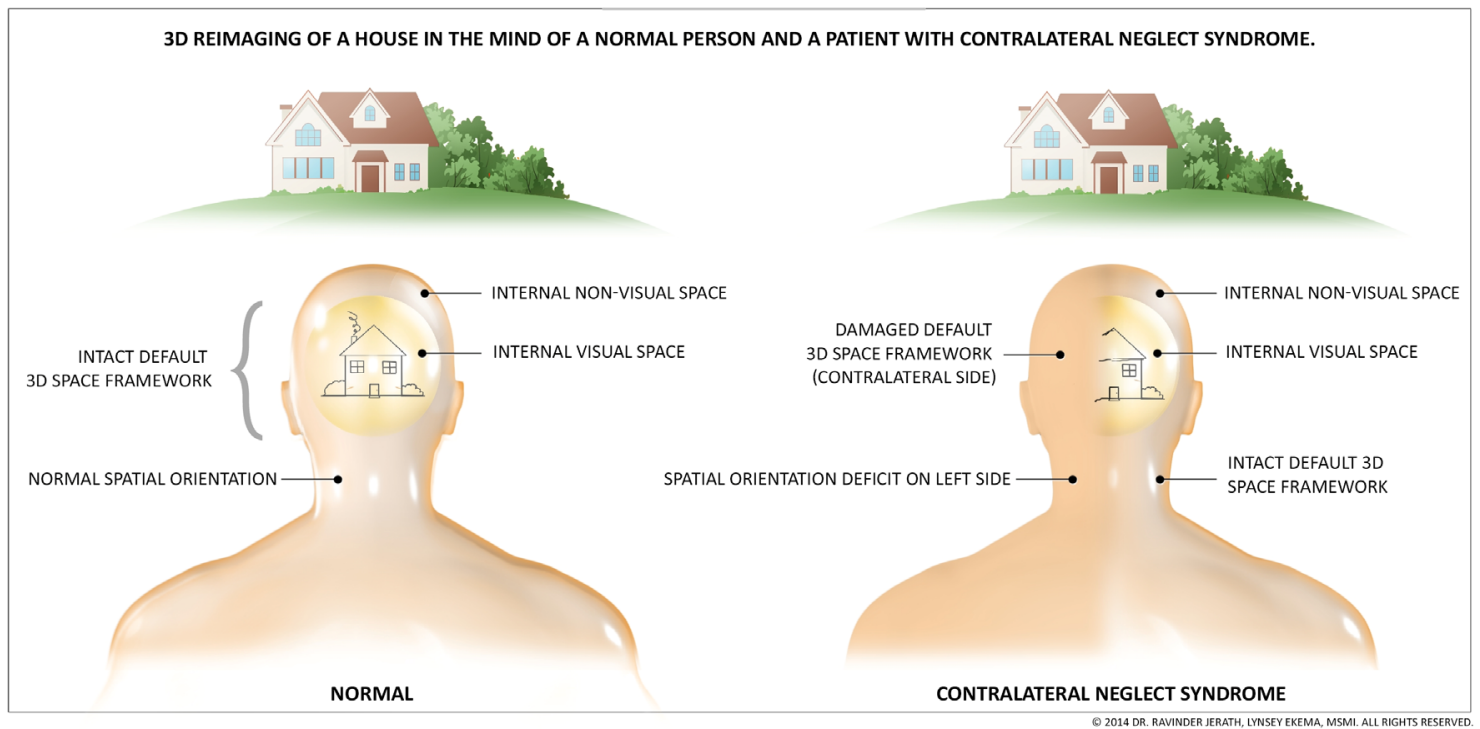

Figure 3. 3D reimaging of a house in the mind of a healthy patient and a patient with contralateral neglect syndrome: in a healthy mind, the image of a house is seen and integrated into the default space. The healthy individual's parietal lobe is fully functional and gives the spatial dimension to visual, non-visual, external, and internal space. In those with contralateral neglect syndrome, the right parietal lobe is damaged. These patients receive visual input to the retina of the entire house; however, sensory information from the left side of sensory fields is lost in the damaged right parietal cortex. When the sensory information of the house is integrated into the default space, all sensory information from the left sensory fields are lost to conscious awareness as the default space requires a spatial framework (Figure by Lynsey Ekema, MSMI) [54]. 
yet they experience an intact, often deformed, painful limb [55]. We have proposed this painful experience is due to an integration conflict between indicators of body schema and an increase in top down neural activity [10]. The amputation may sever incoming information from the periphery; however, the respective central sensory motor cortex is intact and may begin to be overtaken by other areas such as the face and neck [56] (Figure 4). This conflicting, abnormal networking in the CNS results in top down efferent activity from the brain to body to continue at even higher intensities leading to the pain of Phantom Limb Syndrome [10]. Mirror therapy works by resolving the integration conflict between the different sensory inputs [57] for the same limb that are integrated into the default space.

\subsection{Central Pain Syndrome}

Pain and uncomfortable sensations are characteristic of central pain syndrome (Dejerine-Roussy Syndrome) [58]. This syndrome usually develops after injury to one or more nuclei of the thalamus or along the spinothalamic tract [59]. We

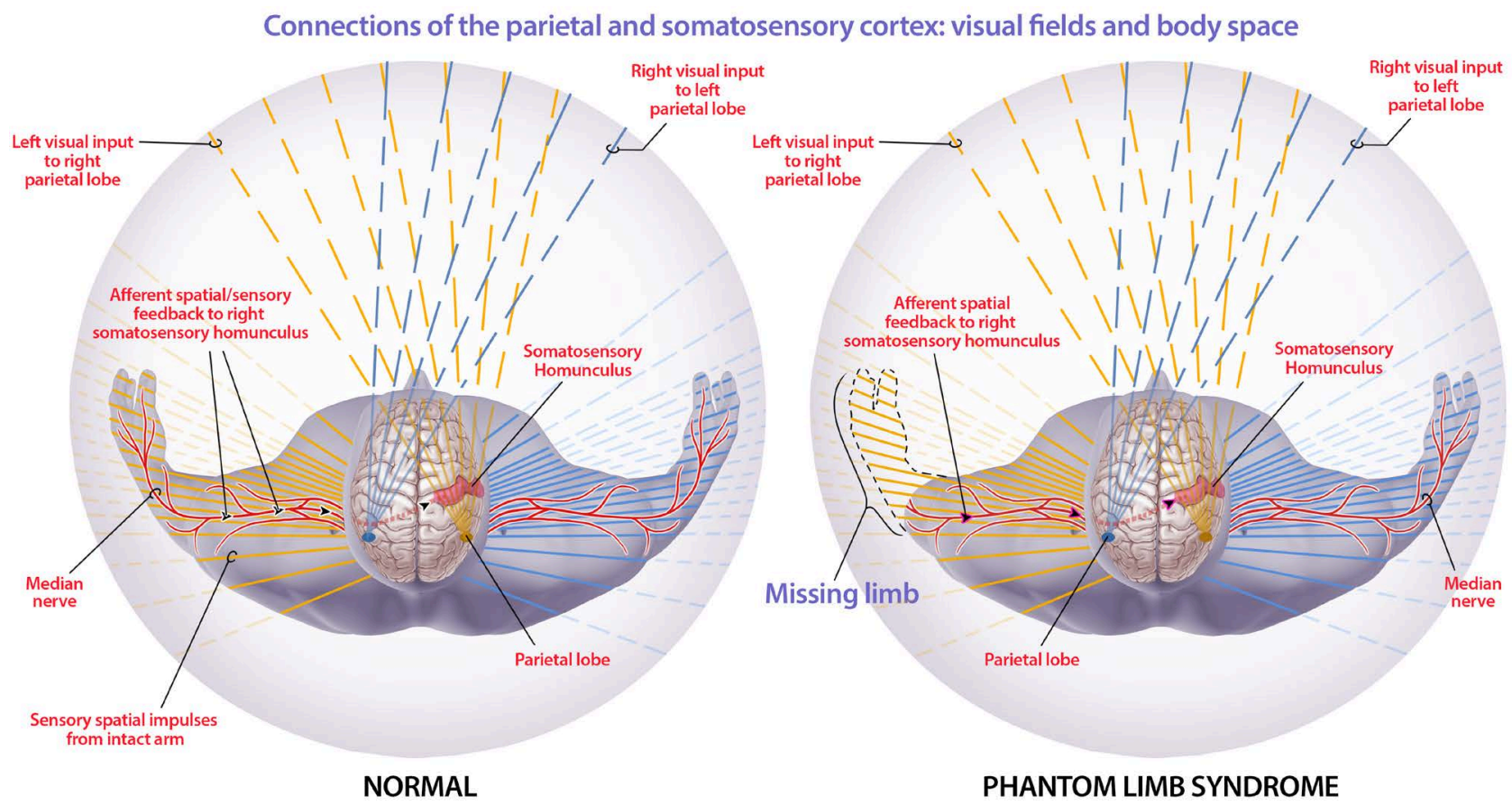

Figure 4. Connections of the parietal and somatosensory cortex: visual fields and body space. The orange lines represent visual and non-visual information sent to the right parietal cortex, and the blue lines represent visual and non-visual information sent to the left parietal cortex. The right sensorimotor cortex is depicted in red with the corresponding red body nerves that illustrate the origin of the sensorimotor information that is filled into the $3 \mathrm{D}$ default space by the thalamus. The figure depicts how the right parietal lobe and sensory motor cortex in a healthy individual receives and processes sensory information from the left side of the body, as well as a patient with a left forearm amputation and subsequent phantom limb syndrome. Visual indication of the missing limb is filled into the $3 \mathrm{D}$ default space so that the missing limb is not included within the internal representation of visual space. The sensorimotor network and other networks in the brain remain intact although they lack afferent input. This leads to the conflict of an intact non-visual representation of the limb with a visual representation of the missing limb, resulting in phantom limb sensations. We assert the integration of this contradictory sensory information and subsequent representation within the $3 \mathrm{D}$ default space result in the painful experience of possessing a phantom limb [5]. 
have proposed that this damage initially results in a discrepancy between the bodily sensory sites, such as the various tactile receptors, and their respective cortical regions [8]. Upon injury, the damage cuts the neural connection between tactile sensory receptors and their cortical sites [60]. Due to the fact that the sensory information is relayed along the spinothalamic tract to the thalamus before it is processed by the cortex [61].

The pain and uncomfortable sensations of the syndrome most often arise over time, and this has been theorized to be due to 1) continuing afferent sensory signals creating new pathways to other undamaged thalamic regions that redirect the information to pain centers and other inappropriate cortical sites [60]; 2) maladaptive homeostatic plasticity [62], and 3) CNS reorganization [63]. Our model allows a full view of the mechanisms of central pain by revealing the circular nature of pain sensation. In this syndrome, the dominant top-down tactile impulses are overridden by the altered CNS activity and alterations described (Figure 5). As these top-down impulses indicating pain and uncomfortable sensations develop over time due to mechanisms listed, the feedback and feedforward loops between the periphery and cortex via the thalamus becomes locked into place in this dysfunctional state and is seen as normal by the corticothalamic network. This condition supports our model by illustrating the top-down nature of sensory perception we have proposed and how its dominance can lead to distorted sensory experiences. We believe this condition may be treated by developing a method to reset the locked network between the periphery and cortex. This may be achieved through virtual reality. If in virtual reality one experiences something similarly painful such as many needles inserted into the painful limb, the brain may identity an external source to the pain experienced and reset the abnormal thalamic tactile network. This effect may work through similar mechanisms to mirror therapy for phantom limb syndrome by resolving a conflict between pain expectation and pain experience [64].

\subsection{Vestibular Disorders and Vertigo}

Many vestibular conditions resulting in a type of dizziness, such as vertigo, show the nature of sensory integration into the default space. Vertigo involves a perceived movement such as swaying or rotation either of one's body, the external space, or both [65]. Strupp and Brandt describe in their 2008 review article a number of vestibular conditions resulting in peripheral vestibular vertigo which is due to damage to the vestibular nerve or organs themselves and result at minimum in disrupted balance and spatial memory [65]. The vestibular conditions they describe and which we explore in citation to their review article include benign paroxysmal positioning vertigo, vestibular neuritis, and Menière's disease. They describe how benign paroxysmal positioning vertigo most commonly results in the sensation of being on a merry-go-round (rotational vertigo) and is due to agglomerates of otoconia that nearly fill the semicircular canal and are freely mobile within it sending erratic sensory information on bodily acceleration. 


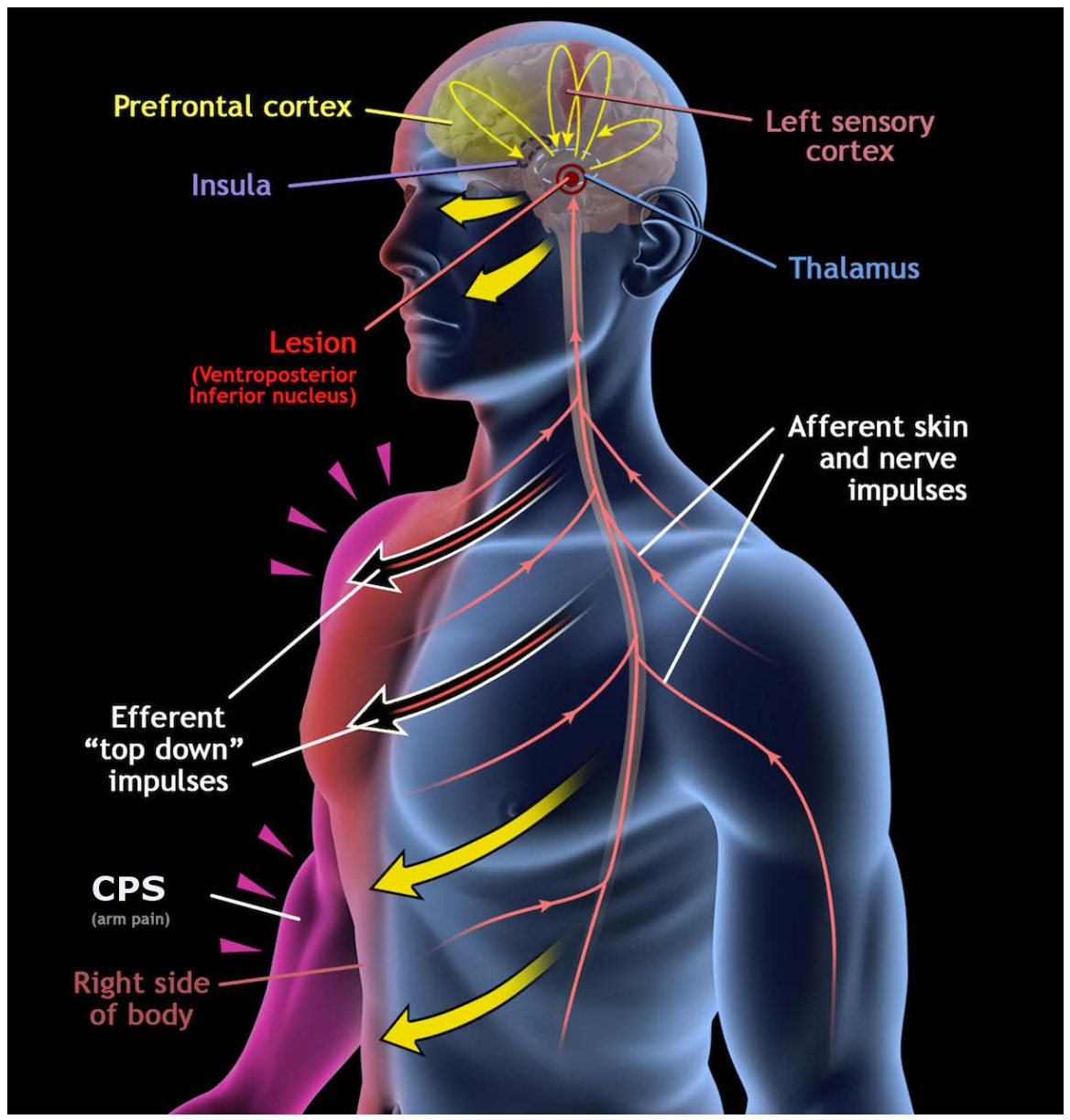

Figure 5. Default Space Theory of Central Pain Syndrome. In patients with central pain, the overriding system of top-down impulses dominates the sensory stream thus leading to dysfunctional cortical networks and subsequent altered sensory information along this efferent projection. In the model, the insula and sensory cortex are hyper-reactive in the presence of normal stimuli, presumably due to maladaptive plasticity and/or the development of alternate neural pathways. Subsequent oscillatory synchronization between receptors and cortex would be dominated by the top-down efferent sensory information. Over time, this leads to the insula and cortex receiving amplified information from the hyperexcitable thalamus along with the development of a thalamic network locked into a state of pain. Previously published in [8].

They also explain vestibular neuritis as a degradation of the vestibular nerve also resulting in rotational vertigo as well as a tendency to fall toward the affected side. Their description of Menière's disease consists of episodes of vestibulocochlear nerve depolarization that produce rotary vertigo and other symptoms. The disruption of the pathways of the vestibular system leading to vertigo highlights the nature of sensory integration into the default space as we have described previously about the dizziness test.

\subsection{Claustrophobia}

Claustrophobia is a condition in which patients have high anxiety or full panic attacks in response to being in or imagining small closed spaces [66]. We have 
proposed a novel etiology of this condition in which the internal space reflects the special characteristics of the external space [51]. Visual sensory input indicates the volume of space surrounding a viewer. The space parameters of the internal space of the default space are primarily set by these visual indications. We have proposed that when the surrounding space of a claustrophobic patient becomes tightly enclosed, this enclosure is reflected into the person's conscious experience which based in the 3D default space and amplifies feelings of being constrained. Similarly, wide expanses of space such as a beach can be reflected into the intra-personal space, creating feelings of freedom. This may be a main reason why the beach is a popular destination for vacationers.

\subsection{Seasonal Affective Disorder}

Seasonal Affective Disorder is a condition in which prolonged states of low external light, such as in extended winters, leads to depression often requiring light therapy and medications [67]. The DST explains how external space is reflected internally as our mind unconsciously develops a replication of external space within its own internal space [11]. One's state of mind is influenced by the internal atmosphere, which is in turn influenced by the external atmosphere. We suggest the knowledge of the nature of this disorder in light of these mechanics can be reassuring to patients who may attribute the depression to uncontrollable life factors, thus leading curative methods to be more successful. Throughout our evolution, we have developed a strong relationship to the sun and when we receive its light, it has great effects on our physiology; most notably through effects on circadian rhythms [68]. These evolutionarily ancient rhythms are reflected in the default space, showing how the default space also has a dimension of time and that it is ancient in its evolutionary origins. Overall, this disorder shows the extent of internal replications of natural environmental patterns of space and time.

\subsection{Grandiose Delusions and Usher's Syndrome}

There exist clinical conditions in which a person has an abnormal self-perception such as narcissism [69]. We propose the intra-personal space of the default space reflects itself strongly and consciously in some predisposed people instead of remaining unconscious. This may result in an overly introspective experience of their internal space. Without an understanding of what they are experiencing, they may confuse the external world with the internal. This may result in beliefs that an infinite external space exists within themselves; therefore they believe they have an omnipotent power over the external world. These sufferers of grandiosity may feel that God or deities are within themselves, or that they are such beings [70]. However, they may not be able to deny their visceral, human nature, awakening them to thoughts that they cannot live up to such grandiose delusions. Reports of low self-esteem, aggressive behavior, or even suicide resulting from these delusions are not uncommon [70]. 
The development of grandiose delusions and the result of these delusions are reflected in Usher's syndrome in which deafness and blindness occur due to genetic abnormality [71]. Sensory deprivation of the syndrome results in patients losing touch with the external world along with minimal social interaction furthering this separation [71]. We assert the default space is still active in these patients, however, lacks visual and auditory sensory input. Therefore, the intra-personal dark space becomes more apparent. 23\% of Usher's syndrome patients develop psychotic symptoms [71], and we propose this overactive introspective view of the default space combined with a lack of understanding of this space can result in one developing grandiose delusions with potentially dangerous behavior.

\subsection{PTSD, Depression, and Deliberate Self-Harm}

We have explored how some mental conditions arise or evolve due to external space defaulting into internal space [9]. In the case of post-traumatic stress disorder (PTSD), patients are tormented by reoccurring vivid memories of past traumatic events [72]. Symptoms include upsetting dreams, thoughts, and feelings about the event as well as avoidance and distress of trauma related cues [72]. Those suffering from PTSD have higher risks of suicide and self-harm [73]. Traumatic events may cause an extreme adrenal response leading to lasting, deep neurological patterns in the brain characteristic of PTSD [74]. A key feature of Complex PTSD is self-destructive behavior [75]. This behavior may arise due to alterations in self-perception [75]. Healing deep rest, counteracting the stress response, experienced during Transcendental Meditation is being used to treat PTSD in active duty Service Members [76]. Cognitive Behavioral Therapy has been shown to be effective in treating PTSD by assisting patients in identifying traumatic memories and thoughts, confronting these, and replacing them with more comforting thoughts [77]. We propose that depression, PTSD, and other conditions potentially leading to self-harm [78] [79] are aggravated by the top-down domination of how the external space is replicated within, as well as the way in which the mind projects the internal atmosphere into how we experience the external atmosphere. Thus, if one has a negative internal atmosphere, they will project this negativity into how the perceive the external world.

Depressive mental conditions that can lead to self-harm may begin with traumatic experiences, unhealthy lifestyles, or psychosis, however, we suggest the resulting introspection into the internal thoughts and feelings of their disease traps patients in their internal worlds, leading their mental conditions to exacerbate in intensity and increased recovery time. The traumatic or depressive thoughts are characteristic of a strongly negative mental atmosphere. This intensely negative internal atmosphere is easily projected into the experience of the external world just as the external world is defaulted into the internal, leading patients to focus on negative qualities of the world and maintain the human existence as negative and futile as reflected from their thought patterns. These pa- 
tients may fail to see the pollution of their internal atmosphere and instead blame the external world for their emotions. We assert that if the DST is accounted for in techniques of behavioral therapy, patients can be taught the nature of their distraught internal perspective. If they can see that the problems they experience are arising from within their own minds, they may gain a more positive perception of the world in which they live, allowing them to overcome the dominating projection of the internal world into the external and allowing the positives of the external world to be reflected into the internal. This may provide motivation to engage in the many social, physical, and mental activities which may bring them happiness.

\section{Conclusions}

The DST is an expansive theory of unified consciousness and cognition. This theory's keystone principle is that the foundation of consciousness is a neural space that replicates the dimensional and other physical qualities of the external environment. This space is filled with sensory information and is formed by the thalamus and processed by corticothalamic oscillatory activity. In this way, the external world is replicated internally by the mind and body. The result is a unified experience of internal and external projections originating from the sensory organs and viscera. Sensations are primed to fall into this live experience allowing a seamless interpretation of our world.

In order to provide support for the DST and allow the layman to understand mechanisms of consciousness and cognition, we have explored numerous observational tests that produce effects that anyone can experience along with phenomenological support from mental conditions with effects on the conscious mind such as contralateral neglect syndrome, claustrophobia, and PTSD. The novelty of this article lies in the nature of deriving physiological and psychological concepts from personal phenomenologies as well as those in mental disorders. We encourage readers to perform the observational tests described in order to better understand the nature of consciousness as described by the model. We predict that if our model is accounted for in the conditions we have described, there will be a significant advancement in the diagnosis and treatment of these conditions.

\section{Acknowledgements}

We would like to thank Dr. Vernon Barnes for editing the manuscript.

\section{Author's Contributions}

Theory developed by RV with some writing with majority of the manuscript written by CB. Images are original art by MJ.

\section{Conflicts of Interest}

The authors declare no conflict of interest. 


\section{References}

[1] Chalmers, D.J. (1995) Facing Up to the Problem of Consciousness. Journal of Consciousness Studies, 2, 200-219.

[2] Harnad, S. (2000) How/Why the Mind-Body Problem Is Hard. Journal of Consciousness Studies, 7, 54-61.

[3] Tononi, G. (2004) An Information Integration Theory of Consciousness. BMC Neurosci, 5, 42. https://doi.org/10.1186/1471-2202-5-42

[4] Baars, B.J. (2005) Global Workspace Theory of Consciousness: Toward a Cognitive Neuroscience of Human Experience. Progress in Brain Research, 150, 45-53. https://doi.org/10.1016/S0079-6123(05)50004-9

[5] Jerath, R. (2015) Connections of the Parietal and Somatosensory Cortex: Visual Fields and Body Space. In: Etiology of Phantom Limb Syndrome: Insights from a 3 D Default Space Consciousness Model, 153-159.

[6] Mcginn, C. (2012) All Machine and No Ghost? New Statesman.

[7] Seth, A.K. (2016) The Real Problem. https://aeon.co/essays/the-hard-problem-of-consciousness-is-a-distraction-from-th e-real-one

[8] Jerath, R., Beveridge, C. and Jensen, M. (2018) Central Pain Syndrome: Etiological Perspectives from the 3D Default Space Model of Consciousness. World Journal of Neuroscience, 8, 277-292. https://doi.org/10.4236/wjns.2018.82022

[9] Jerath, R. and Crawford, M.W. (2014) Neural Correlates of Visuospatial Consciousness in 3D Default Space: Insights from Contralateral Neglect Syndrome. Consciousness and Cognition, 28, 81-93. https://doi.org/10.1016/j.concog.2014.06.008

[10] Jerath, R., Crawford, M.W. and Jensen, M. (2015) Etiology of Phantom Limb Syndrome: Insights from a 3D Default Space Consciousness Model. Medical Hypotheses, 85, 153-159. https://doi.org/10.1016/j.mehy.2015.04.025

[11] Jerath, R., Crawford, M.W. and Barnes, V.A. (2015b) A Unified 3D Default Space Consciousness Model Combining Neurological and Physiological Processes That Underlie Conscious Experience. Frontiers in Psychology, 6, 1204.

[12] Fingelkurts, A.A., Fingelkurts, A.A. and Neves, C.F.H. (2010) Natural World Physical, Brain Operational, and Mind Phenomenal Space-Time. Physics of Life Reviews, 7, 195-249. https://doi.org/10.1016/j.plrev.2010.04.001

[13] Oizumi, M., Albantakis, L. and Tononi, G. (2014) From the Phenomenology to the Mechanisms of Consciousness: Integrated Information Theory 3.0. PLoS Computational Biology, 10, e1003588. https://doi.org/10.1371/journal.pcbi.1003588

[14] Revonsuo, A. (2006) Inner Presence: Consciousness as a Biological Phenomenon. MIT Press, Cambridge.

[15] Greene, B. (2017) How Does Consciousness Happen? TED Conferences. https://blog.ted.com/how-does-consciousness-happen-anil-seth-speaks-at-ted2017/

[16] Jerath, R., Cearley, S.M., Barnes, V.A. and Junca, S. (2017) The Dynamic Role of Breathing and Cellular Membrane Potentials in the Experience of Consciousness. World Journal of Neuroscience, 7, 66-81. https://doi.org/10.4236/wjns.2017.71007

[17] Edelman, G.M., Gally, J.A. and Baars, B.J. (2011) Biology of Consciousness. Frontiers in Psychology, 2, 4. https://doi.org/10.3389/fpsyg.2011.00004

[18] Mader, S.S. (2000) Human Biology. McGraw-Hill, New York.

[19] Jerath, R., Cearley, S.M., Barnes, V.A. and Nixon-Shapiro, E. (2016) How Lateral 
Inhibition and Fast Retinogeniculo-Cortical Oscillations Create Vision: A New Hypothesis. Medical Hypotheses, 96, 20-29. https://doi.org/10.1016/j.mehy.2016.09.015

[20] Jerath, R., Cearley, S.M., Paladiya, R. and Barnes, V.A. (2017) Sensory Consciousness Is Experienced through Amplification of Sensory Stimuli via Lateral Inhibition. World Journal of Neuroscience, 7, 244-256. https://doi.org/10.4236/wjns.2017.73020

[21] Jerath, R. and Beveridge, C. (2018) Top Mysteries of the Mind: Insights from the Default Space Model of Consciousness. Frontiers in Human Neuroscience, 12, 162. https://doi.org/10.3389/fnhum.2018.00162

[22] Yantis, S. (2014) Sensation and Perception. Worth Publishers, New York.

[23] Wolinsky, H. (2008) Paths to Acceptance. The Advancement of Scientific Knowledge Is an Uphill Struggle against “Accepted Wisdom”. EMBO Reports, 9, 416-418. https://doi.org/10.1038/embor.2008.65

[24] Costantini, M. and Haggard, P. (2007) The Rubber Hand Illusion: Sensitivity and Reference Frame for Body Ownership. Consciousness and Cognition, 16, 229-240. https://doi.org/10.1016/j.concog.2007.01.001

[25] Tsakiris, M. and Haggard, P. (2005) The Rubber Hand Illusion Revisited: Visuotactile Integration and Self-Attribution. Journal of Experimental Psychology: Human Perception and Performance, 31, 80-91. https://doi.org/10.1037/0096-1523.31.1.80

[26] Salomon, R., Lim, M., Pfeiffer, C., Gassert, R. and Blanke, O. (2013) Full Body Illusion Is Associated with Widespread Skin Temperature Reduction. Frontiers in Behavioral Neuroscience, 7, 65.

[27] Moseley, G.L., Olthof, N., Venema, A., Don, S., Wijers, M., Gallace, A. and Spence, C. (2008) Psychologically Induced Cooling of a Specific Body Part Caused by the Illusory Ownership of an Artificial Counterpart. Proceedings of the National Academy of Sciences of the United States of America, 105, 13169-13173. https://doi.org/10.1073/pnas.0803768105

[28] Jerath, R., Crawford, M.W. and Barnes, V.A. (2015) Functional Representation of Vision within the Mind: A Visual Consciousness Model Based in 3D Default Space. Journal of Medical Hypotheses and Ideas, 9, 46-56.

[29] Spoor, F., Garland, T., Krovitz, G., Ryan, T.M., Silcox, M.T. and Walker, A. (2007) The Primate Semicircular Canal System and Locomotion. Proceedings of the National Academy of Sciences of the United States of America, 104, 10808-10812. https://doi.org/10.1073/pnas.0704250104

[30] Brindley, G.S. (1962) Two New Properties of Foveal After-Images and a Photochemical Hypothesis to Explain Them. The Journal of Physiology, 164, 168-179. https://doi.org/10.1113/jphysiol.1962.sp007011

[31] Shimojo, S., Kamitani, Y. and Nishida, S. (2001) Afterimage of Perceptually Filled-In Surface. Science, 293, 1677-1680. https://doi.org/10.1126/science.1060161

[32] Holcombe, A.O., Macleod, D.I.A. and Mitten, S.T. (2004) Positive Afterimages Caused by a Filled-In Representation. Journal of Vision, 4, 485.

https://doi.org/10.1167/4.8.485

[33] Sperandio, I., Lak, A. and Goodale, M.A. (2012) Afterimage Size Is Modulated by Size-Contrast Illusions. Journal of Vision, 12, 18. https://doi.org/10.1167/12.2.18

[34] Jerath, R., Crawford, M.W. and Barnes, V.A. (2015) Functional Representation of Vision within the Mind: A Visual Consciousness Model Based in 3D Default Space. Journal of Medical Hypotheses and Ideas, 9, 45-56. 
https://doi.org/10.1016/j.jmhi.2015.02.001

[35] Gersztenkorn, D. and Lee, A.G. (2015) Palinopsia Revamped: A Systematic Review of the Literature. Survey of Ophthalmology, 60, 1-35. https://doi.org/10.1016/j.survophthal.2014.06.003

[36] Jerath, R.C., S.M., Barnes, V.A. and Jensen, M. (2018) Micro-Calibration of Space and Motion by Photoreceptors Synchronized in Parallel with Cortical Oscillations: A Unified Theory of Visual Perception. Medical Hypotheses, 110, 71-75. https://doi.org/10.1016/j.mehy.2017.11.005

[37] Clarke, T., Black, L., Stussman, B., Barnes, P. and Nahin, R. (2015) Trends in the Use of Complementary Health Approaches among Adults: United States, 2002-2012. National Health Statistics Reports, National Center for Health Statistics, Hyattsville, MD.

[38] Singleton, O., Holzel, B.K., Vangel, M., Brach, N., Carmody, J. and Lazar, S.W. (2014) Change in Brainstem Gray Matter Concentration Following a Mindfulness-Based Intervention Is Correlated with Improvement in Psychological Well-Being. Frontiers in Human Neuroscience, 8, 33. https://doi.org/10.3389/fnhum.2014.00033

[39] Deshmukh, V.D. (2006) Neuroscience of Meditation. Scientific World Journal, 6, 2239-2253. https://doi.org/10.1100/tsw.2006.353

[40] Travis, F. (2014) Transcendental Experiences during Meditation Practice. Annals of the New York Academy of Sciences, 1307, 1-8. https://doi.org/10.1111/nyas.12316

[41] Berkovich-Ohana, A., Dor-Ziderman, Y., Glicksohn, J. and Goldstein, A. (2013) Alterations in the Sense of Time, Space, and Body in the Mindfulness-Trained Brain: A Neurophenomenologically-Guided MEG Study. Frontiers in Psychology, 4, 912. https://doi.org/10.3389/fpsyg.2013.00912

[42] Jerath, R., Cearley, S.M., Barnes, V.A. and Jensen, M. (2016) Meditation Experiences, Self, and Boundaries of Consciousness. International Journal of Complementary \& Alternative Medicine, 4, Article ID: 00105.

[43] Venkatesh, S., Raju, T.R., Shivani, Y., Tompkins, G. and Meti, B.L. (1997) A Study of Structure of Phenomenology of Consciousness in Meditative and Non-Meditative States. Indian Journal of Physiology and Pharmacology, 41, 149-153.

[44] Simons, D. (2010) Selective Attention Test [Video File].

[45] Chabris, C.F. and Simons, D. (2010) The Invisible Gorilla. Harmony.

[46] Jerath, R. and Crawford, M.W. (2015) Layers of Human Brain Activity: A Functional Model Based on the Default Mode Network and Slow Oscillations. Frontiers in Human Neuroscience, 9, 248. https://doi.org/10.3389/fnhum.2015.00248

[47] Metzger, W. (1930) Optische Untersuchungen am Ganzfeld [The First Psychophysiological Study with Regard to Ganzfelds]. Psychologische Forschung, 13, 6-29.

[48] Sireteanu, R., Oertel, V., Mohr, H., Linden, D. and Singer, W. (2008) Graphical Illustration and Functional Neuroimaging of Visual Hallucinations during Prolonged Blindfolding: A Comparison to Visual Imagery. Perception, 37, 1805-1821. https://doi.org/10.1068/p6034

[49] Jeffery, K. (1985) The Divided Province: The Troubles in Northern Ireland 1969-1985. Orbis, London.

[50] Wackermann, J., Pütz, P. and Allefeld, C. (2008) Ganzfeld-Induced Hallucinatory Experience, Its Phenomenology and Cerebral Electrophysiology. Cortex, 44, 1364-1378. https://doi.org/10.1016/j.cortex.2007.05.003

[51] Jerath, R., Harden, K., Crawford, M., Barnes, V.A. and Jensen, M. (2014) Role of 
Cardiorespiratory Synchronization and Sleep Physiology: Effects on Membrane Potential in the Restorative Functions of Sleep. Sleep Medicine, 15, 279-288.

https://doi.org/10.1016/j.sleep.2013.10.017

[52] Kerkhoff, G. (2001) Spatial Hemineglect in Humans. Progress in Neurobiology, 63, 1-27. https://doi.org/10.1016/S0301-0082(00)00028-9

[53] Jerath, R. and Crawford, M.W. (2014) Neural Correlates of Visuospatial Consciousness in 3D Default Space: Insights from Contralateral Neglect Syndrome. Consciousness and Cognition, 28, 81-93.

https://doi.org/10.1016/j.concog.2014.06.008

[54] Jerath, R. and Ekema, L. (2014) 3D Reimaging of a House in the Mind of a Healthy Patient and a Patient with Contralateral Neglect Syndrome. In: Neural Correlates of Visuospatial Consciousness in $3 D$ Default Space: Insights from Contralateral Neglect Syndrome, 81-93.

[55] Weeks, S.R., Anderson-Barnes, V.C. and Tsao, J.W. (2010) Phantom Limb Pain: Theories and Therapies. Neurologist, 16, 277-286. https://doi.org/10.1097/NRL.0b013e3181edf128

[56] Ramachandran, V.S. and Hirstein, W. (1998) The Perception of Phantom Limbs. The D. O. Hebb Lecture. Brain, 121, 1603-1630. https://doi.org/10.1093/brain/121.9.1603

[57] Ramachandran, V.S. and Altschuler, E.L. (2009) The Use of Visual Feedback, in Particular Mirror Visual Feedback, in Restoring Brain Function. Brain, 132, 1693-1710. https://doi.org/10.1093/brain/awp135

[58] Wilton, L.M. (1989) Thalamic Pain Syndrome. Journal of Neuroscience Nursing, 21, 362-365. https://doi.org/10.1097/01376517-198912000-00007

[59] Sherman, S.M. and Guillery, R.W. (2001) Exploring the Thalamus. Academic Press, San Diego.

[60] Schott, B., Laurent, B. and Mauguiere, F. (1986) Thalamic Pain: Critical Study of 43 Cases. Revue Neurologique (Paris), 142, 308-315.

[61] Herrero, M.T., Barcia, C. and Navarro, J.M. (2002) Functional Anatomy of Thalamus and Basal Ganglia. Child s Nervous System, 18, 386-404. https://doi.org/10.1007/s00381-002-0604-1

[62] Wang, G. and Thompson, S.M. (2008) Maladaptive Homeostatic Plasticity in a Rodent Model of Central Pain Syndrome: Thalamic Hyperexcitability after Spinothalamic Tract Lesions. The Journal of Neuroscience, 28, 11959-11969. https://doi.org/10.1523/JNEUROSCI.3296-08.2008

[63] Lenz, F.A., Garonzik, I.M., Baker, F.H., Richardson, R.T. and Dougherty, P.M. (1998) Reorganization of Sensory Modalities Evoked by Microstimulation in Region of the Thalamic Principal Sensory Nucleus in Patients with Pain Due to Nervous System Injury. Journal of Comparative Neurology, 14, 125-138. https://doi.org/10.1002/(SICI)1096-9861(19980914)399:1<125::AID-CNE10>3.0.CO ;2-G

[64] Kim, S.Y. and Kim, Y.Y. (2012) Mirror Therapy for Phantom Limb Pain. The Korean Journal of Pain, 25, 272-274. https://doi.org/10.3344/kjp.2012.25.4.272

[65] Strupp, M. and Brandt, T. (2008) Diagnosis and Treatment of Vertigo and Dizziness. Deutsches Ärzteblatt International, 105, 173-180.

[66] Ost, L.G. (2007) The Claustrophobia Scale: A Psychometric Evaluation. Behaviour Research and Therapy, 45, 1053-1064. https://doi.org/10.1016/j.brat.2004.10.004

[67] Lam, R.W., Levitt, A.J., Levitan, R.D., Enns, M.W., Morehouse, R., Michalak, E.E. 
and Tam, E.M. (2006) The Can-SAD Study: A Randomized Controlled Trial of the Effectiveness of Light Therapy and Fluoxetine in Patients with Winter Seasonal Affective Disorder. The American Journal of Psychiatry, 163, 805-812. https://doi.org/10.1176/ajp.2006.163.5.805

[68] Edgar, R.S., Green, E.W., Zhao, Y., Van Ooijen, G., Olmedo, M., Qin, X., Xu, Y., Pan, M. and Valekunja, U.K. (2015) Peroxiredoxins Are Conserved Markers of Circadian Rhythms. Nature, 485, 459-464. https://doi.org/10.1038/nature11088

[69] Caligor, E., Levy, K.N. and Yeomans, F.E. (2015) Narcissistic Personality Disorder: Diagnostic and Clinical Challenges. The American Journal of Psychiatry, 172, 415-422. https://doi.org/10.1176/appi.ajp.2014.14060723

[70] Joseph, Z., Victor, K. and Rimona, D. (2011) "Ego-Dystonic" Delusions as a Predictor of Dangerous Behavior. Psychiatric Quarterly, 82, 113-120.

https://doi.org/10.1007/s11126-010-9150-2

[71] Viala, A., Nicot, T., Levy, F. and Vacheron, M.N. (2009) À propos d'un cas de syndrome de Usher suivi en psychiatrie: Intérêt du diagnostic somatique pour la prise en charge psychiatrique. L'Encéphale, 35, 286-291.

https://doi.org/10.1016/j.encep.2008.04.002

[72] Association, A.P. (2013) Diagnostic and Statistical Manual of Mental Disorders. American Psychiatric Publishing, Arlington, VA. https://doi.org/10.1176/appi.books.9780890425596

[73] Bisson, J., Cosgrove, S., Lewis, C. and Robert, N. (2015) Post-Traumatic Stress Disorder. $B M J, 351$, h6161.

[74] Rothschild, B. (2000) The Body Remembers: The Psychophysiology of Trauma and Trauma Treatment. W. W. Norton \& Company, New York.

[75] Dyer, K.F.W., Dorahy, M.J., Hamilton, G., Corry, M., Shannon, M., Macsherry, A., Mcrobert, G., Elder, R. and Mcelhill, B. (2009) Anger, Aggression, and Self-Harm in PTSD and Complex PTSD. Journal of Clinical Psychology, 65, 1099-1114. https://doi.org/10.1002/jclp.20619

[76] Barnes, V.A., Monto, A., Williams, J.J. and Rigg, J.L. (2016) Impact of Transcendental Meditation on Psychotropic Medication Use among Active Duty Military Service Members with Anxiety and PTSD. Military Medicine, 181, 56-63. https://doi.org/10.7205/MILMED-D-14-00333

[77] Mulick, P.S., Landes, S.J. and Kanter, J.W. (2005) Contextual Behavior Therapies in the Treatment of PTSD: A Review. International Journal of Behavioral Consultation and Therapy, 1, 223-228. https://doi.org/10.1037/h0100747

[78] Gratz, K.L. and Tull, M.T. (2012) Exploring the Relationship between Posttraumatic Stress Disorder and Deliberate Self-Harm: The Moderating Roles of Borderline and Avoidant Personality Disorders. Psychiatry Research, 199, 19-23. https://doi.org/10.1016/j.psychres.2012.03.025

[79] Zubrick, S.R., Hafekost, J., Johnson, S.E., Sawyer, M.G., Patton, G. and Lawrence, D. (2017) The Continuity and Duration of Depression and Its Relationship to Non-Suicidal Self-Harm and Suicidal Ideation and Behavior in Adolescents 12-17. Journal of Affective Disorders, 220, 49-56. https://doi.org/10.1016/j.jad.2017.05.050 